\title{
Differential Effects of Mental Stress on Plasma Homovanillic Acid in Schizophrenia and Normal Controls
}

\author{
Tomiki Sumiyoshi, M.D., Ph.D., Osamu Saitoh, M.D., Ph.D., Takashi Yotsutsuji, M.D., Ph.D., \\ Hiroko Itoh, B.C., Kenzo Kurokawa, M.D., Ph.D., and Masayoshi Kurachi, M.D., Ph.D.
}

\begin{abstract}
We previously reported that mental stress by Kraepelin's arithmetic test decreases plasma homovanillic acid ( $p H V A$ ) levels in psychiatrically normal healthy human subjects. The present study was undertaken to determine whether this pattern of changes in $p H V A$ concentrations resulting from mental stress is altered in patients with schizophrenia. Fourteen male patients with schizophrenia including those under ongoing neuroleptic treatment and 14 normal male volunteers participated in the study. Following overnight fast and restricted physical activity, the subjects performed Kraepelin's arithmetic test for 30 minutes. Plasma samples were collected immediately before and after the test for measurement of $\mathrm{pHVA}$ levels. A significant diagnosis by Kraepelin's test effect was observed due to a decrease in
\end{abstract}

pHVA levels by the Kraepelin test in control subjects but not in patients with schizophrenia. Changes in $p H V A$ levels during the Kraepelin test positively correlated with pre-test $p H V A$ levels in control subjects, while this correlation was not observed in patients with schizophrenia. These results may be further support for the presence of a dopamine-dependent restitutive system in the brain. The absence of response of $p H V A$ levels to mental stress in patients with schizophrenia may indicate that the dopamine restitutive system in these patients is disrupted or already down-regulated, as previously predicted.

[Neuropsychopharmacology 20:365-369, 1999]

(C) 1999 American College of Neuropsychopharmacology.

Published by Elsevier Science Inc.
KEY WORDS: Plasma homovanillic acid; $p H V A$; Kraepelin test; Mental stress; Schizophrenia; Dopamine restitutive system

The relevance of dopamine (DA) to the pathophysiology of schizophrenia has been extensively documented (e.g., Reynolds 1983; Seeman et al. 1993; Wong et al. 1986). Despite some argument (Kopin et al. 1988; Lambert et al. 1993; Maas et al. 1988), several lines of investi-

From the Department of Neuropsychiatry, Toyama Medical and Pharmaceutical University, School of Medicine, 2630 Sugitani, Toyama 930-0194 Japan.

Address correspondence to: Tomiki Sumiyoshi, M.D., Ph.D., Department of Neuropsychiatry, Toyama Medical and Pharmaceutical University, School of Medicine, 2630 Sugitani, Toyama, 9300194 Japan.

Received February 26, 1998; revised July 10, 1998; accepted July 20, 1998 gations have focused on plasma levels of homovanillic acid (pHVA), a major metabolite of DA, for the study of DA-related mental disorders such as schizophrenia based on the assumption that dysfunction in central dopaminergic activity is, to some extent, reflected in this peripheral measure (see Friedhoff and Amin 1997 for review). While conflicting results have been reported regarding differences in pHVA levels between patients with schizophrenia and control subjects (Doran et al. 1985; Koreen et al. 1994; Maas et al. 1993; Pickar et al. 1984; Steinberg et al. 1993; Sumiyoshi et al. 1997a; Whelton et al. 1993), there has been accumulated evidence for the association between pHVA levels in schizophrenia and the outcome of neuroleptic treatment (Akiyama et al. 1995; Chang et al. 1993; Davis et al. 1985; Garver et al. 1997; Green et al. 1993; Nagaoka et al. 1997; Sumiyoshi et al. 1997b). 
The presence of a DA-dependent protective system in the brain to maintain mental stability has been proposed (Friedhoff 1985, 1986, 1988). Friedhoff (1988) has hypothesized that a physiological function of the dopaminergic system is to make the brain more resistant to psychotic destabilization by decreasing its own activity in the face of stress, that is reflected in the change of pHVA levels. This hypothesis of the dopaminergic buffer system or the "DA-dependent restitutive system" (Friedhoff 1985, 1986, 1988) may be supported by our recent observation indicating a decrease in pHVA levels during the Kraepelin arithmetic test in psychiatrically normal healthy volunteers (Sumiyoshi et al. 1997c, 1998).

According to the DA-dependent restitutive system theory, the psychotic symptoms of schizophrenia may occur in an individual when (1) there are deficiencies in the function of this system itself which normally downregulates adequately in the face of psychotogenic factors (environmental, psychological, genetic, or physiological) to prevent developing psychosis, or (2) these precipitating psychotogenic factors are so persistent that even adequate dopaminergic compensation (downregulation) is overridden (Friedhoff 1986). In the former case, administration of neuroleptic drugs that are DA receptor blockers would down-regulate the system leading to symptomatic improvement (Friedhoff 1986). Therefore, it is speculated that blunted dopaminergic response to stress represented by pHVA levels would be observed in patients with schizophrenia, independent of whether they are neuroleptic-naive or already receiving neuroleptic treatment, due to a disrupted or already down-regulated DA-dependent restitutive system.
The aim of the present study was to test the hypothesis that the decrease in pHVA levels due to mental stress resulting from the Kraepelin arithmetic test (Sumiyoshi et al. 1997c, 1998) would be blunted in patients with schizophrenia. The study design employed both neuroleptic-naive patients with schizophrenia and those under ongoing neuroleptic treatment, as it is expected from the above considerations that blunted dopaminergic response to stress would be observed across these patient groups.

\section{SUBJECTS AND METHODS}

Fourteen male inpatients with schizophrenia who met DSM-IV criteria for schizophrenia or schizophreniform disorder and 14 male psychiatrically normal healthy volunteers participated in the study. Diagnosis was based on structured interview by experienced psychiatrists and medical history. All subjects gave written consent before entry to the study. Each had a physical and neurological examination, as well as a variety of standard laboratory tests to screen for medical illness. Demographic data of these subjects are presented in Table 1 . Seven patients were under ongoing neuroleptic treatment that had started at least 6 months before. These patients received the last neuroleptic administrations $12 \mathrm{~h}$ before taking the Kraepelin test. The rest of the patients were neuroleptic-naive. Physical exercise, highmonoamine diets, alcohol, and caffeine were restricted for all subjects from the entry to the study $(>24 \mathrm{~h}$ prior to blood sampling).

Table 1. Plasma Homovanillic Acid Levels (ng/ml) before and after Kraepelin's Arithmetic Test

\begin{tabular}{|c|c|c|c|c|c|c|c|c|c|}
\hline \multicolumn{4}{|c|}{ Controls } & \multicolumn{4}{|c|}{ Schizophrenia } & \multirow[b]{2}{*}{ Drug Dose $^{a}$} & \multirow[b]{2}{*}{ Duration of Illness } \\
\hline & Age (yr) & Pre-Test & Post-Test & & Age (yr) & Pre-Test & Post-Test & & \\
\hline a & 20 & 6.59 & 5.79 & A & 16 & 3.54 & 3.58 & naive & $1.5 \mathrm{yr}$ \\
\hline $\mathrm{b}$ & 24 & 8.08 & 7.20 & $\mathrm{~B}$ & 19 & 4.87 & 6.68 & naive & $2 \mathrm{mo}$ \\
\hline c & 25 & 11.38 & 9.76 & $\mathrm{C}$ & 21 & 4.65 & 4.70 & naive & $2 \mathrm{yr}$ \\
\hline $\mathrm{d}$ & 26 & 6.61 & 6.10 & $\mathrm{D}$ & 22 & 5.84 & 5.48 & 6.2 & $5 \mathrm{yr}$ \\
\hline $\mathrm{e}$ & 27 & 6.99 & 6.76 & $\mathrm{E}$ & 22 & 10.76 & 11.97 & naive & $6 \mathrm{mo}$ \\
\hline $\mathrm{f}$ & 28 & 6.55 & 6.36 & $\mathrm{~F}$ & 25 & 7.76 & 7.72 & $18<$ & $7 \mathrm{yr}$ \\
\hline $\mathrm{g}$ & 28 & 7.20 & 6.93 & G & 25 & 7.94 & 8.05 & naive & $1 \mathrm{yr}$ \\
\hline h & 29 & 6.12 & 6.32 & $\mathrm{H}$ & 29 & 5.18 & 4.82 & naive & $13 \mathrm{yr}$ \\
\hline $\mathrm{i}$ & 30 & 6.76 & 6.86 & I & 30 & 6.20 & 5.19 & 2 & $4 \mathrm{yr}$ \\
\hline j & 30 & 8.24 & 6.77 & $\mathrm{~J}$ & 30 & 10.78 & 9.33 & naive & $10 \mathrm{mo}$ \\
\hline $\mathrm{k}$ & 31 & 3.89 & 3.36 & $\mathrm{~K}$ & 31 & 7.69 & 7.25 & 9.8 & $3 \mathrm{yr}$ \\
\hline 1 & 31 & 6.14 & 5.95 & $\mathrm{~L}$ & 38 & 10.37 & 10.17 & 5.7 & $20 \mathrm{yr}$ \\
\hline $\mathrm{m}$ & 34 & 8.78 & 6.78 & $\mathrm{M}$ & 40 & 5.23 & 5.36 & 8 & $13 \mathrm{yr}$ \\
\hline $\mathrm{n}$ & 34 & 6.98 & 6.63 & $\mathrm{~N}$ & 51 & 8.95 & 10.20 & 10 & $25 \mathrm{yr}$ \\
\hline Mean & 28.4 & 7.16 & $6.54^{b}$ & & 28.5 & 7.12 & 7.18 & & \\
\hline SE & 1.0 & 0.45 & 0.35 & & 2.5 & 0.65 & 0.67 & & \\
\hline
\end{tabular}

${ }^{a}$ Drug doses are expressed as haloperidol equivalent (mg/day).

${ }^{b}$ Significantly different from pre-test value $(p<.005$ by two-tailed paried $t$-test). 
The Kraepelin test and blood sampling procedure was adopted from our previous report (Sumiyoshi et al. 1998). Briefly, the subjects underwent the pre-test blood sampling through an intravenous line, that had been obtained at least $30 \mathrm{~min}$ before, at 9:00 AM on the day of investigation following an overnight fast. The subjects rested after the venous line was inserted. Immediately after the pre-test blood sampling, the subjects performed a modified version of Kraepelin's arithmetic test (Kuraishi et al. 1957) for $30 \mathrm{~min}$. On termination of the Kraepelin test (30 min after the pre-test blood sampling), blood samples were again collected for measurement of the post-test pHVA levels. Plasma was separated by centrifugation and stored at $-80^{\circ} \mathrm{C}$ until the free HVA was measured.

Plasma HVA analysis was carried out by high-performance liquid chromatography (HPLC) with coulometric detection using d.l.-Isoproterenol as internal standard (Sumiyoshi et al. 1998). The coefficients of variation $(\mathrm{CV})$ for within-day $(n=6)$ and between-day $(n=$ 6 ) analyses of pHVA were $2.4 \%$ and $3.3 \%$, respectively.

Repeated measures analysis of variance (ANOVA) were performed to determine the diagnosis (schizophrenia or control subjects) by Kraepelin's test effect between these two groups. Pre- and post-Kraepelin test pHVA levels in each group were compared by twotailed paired $t$-test. Spearman's correlation coefficients were obtained relating pre-test $\mathrm{pHVA}$ levels with changes in pHVA values (calculated by subtracting post-test pHVA levels from pre-test pHVA levels) in each group. Differences were considered to be significant when $p$-values were less than 05 .

\section{RESULTS}

Plasma HVA levels before and after the Kraepelin test in each subject are presented in Table 1 . There was no significant correlation between age vs. pre- or post-test pHVA levels both within and across the patient or control groups (data not presented). In subjects with schizophrenia, the pre- or post-test pHVA levels of 7 patients under ongoing neuroleptic treatment did not differ significantly from those of 7 neuroleptic-naive patients $(F(1,12)=.21, p=.65$ and $F(1,12)=.053, p=.82$ by ANOVA, respectively). Neuroleptic doses did not correlate significantly with pre- or post-test pHVA levels in 7 patients receiving neuroleptic treatment (data not shown). Repeated measurers ANOVA revealed a significant diagnosis by Kraepelin's test effect $(F(1,26)=$ $5.35, p<.03)$, accompanied with no significant effect of diagnosis $(F(1,26)=.16, p=.70)$. In control subjects, a significant decrease in pHVA levels due to the Kraepelin test was observed $(t=3.54, p<.005)$, while pHVA levels in patients with schizophrenia were not affected by the Kraepelin test $(t=-.22, p=.83)$.
Figure 1 demonstrates the relation between pre-test pHVA levels and the value of changes in pHVA levels following the Kraepelin test. Changes in pHVA levels during the Kraepelin test positively correlated with pretest pHVA levels in control subjects $\left(r_{\mathrm{s}}=.66, p<.02\right)$, while this correlation was not observed in patients with schizophrenia $\left(r_{\mathrm{s}}=.15, p=.58\right)$. The value of changes in pHVA levels following the Kraepelin test did not correlate significantly with age in control subjects, schizophrenic patients, or the combined population (data not shown). No significant difference was found in the value of changes in pHVA levels between neurolepticnaive patients and those receiving neuroleptic treatment $(F(1,12)=.38, p=.55)$. Neuroleptic doses did not correlate with the value of change in pHVA levels following the Kraepelin test in 7 patients receiving neuroleptic treatment (data not shown).
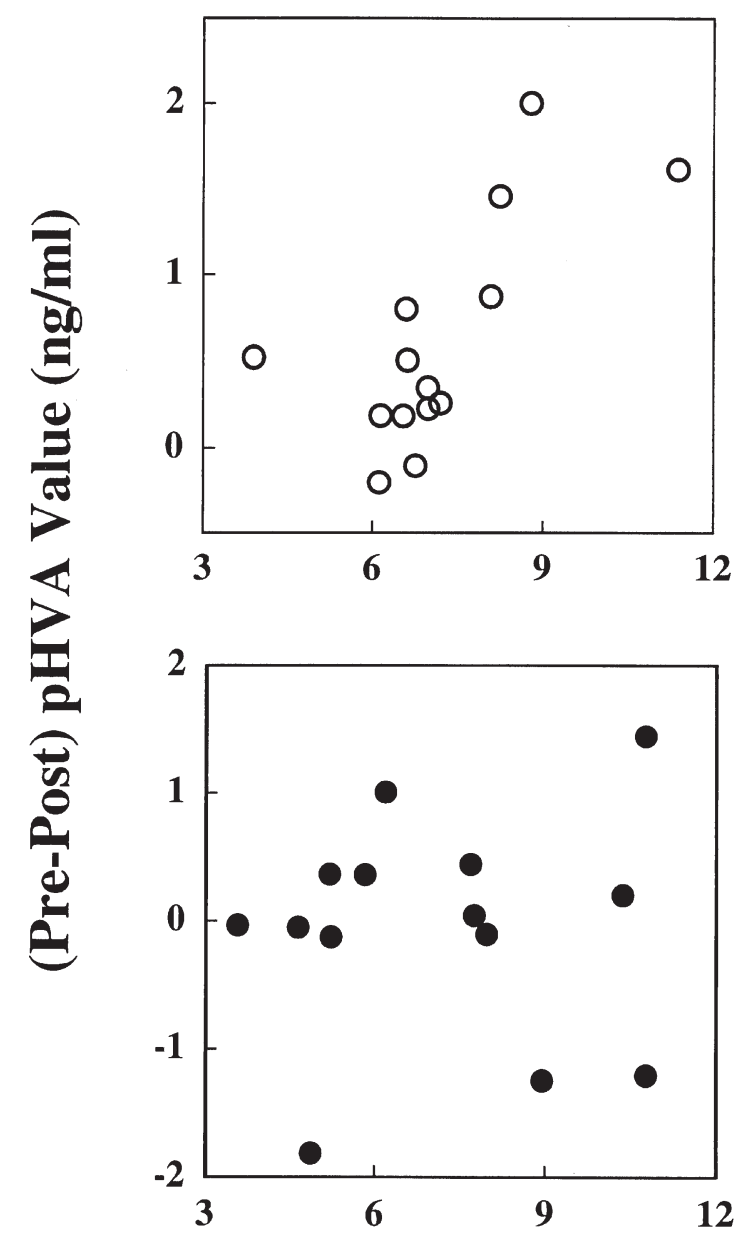

\section{Pre-test pHVA Levels (ng/ml)}

Figure 1. Correlations between the baseline (pre-test) pHVA levels and the value of changes in pHVA levels during the Kraepelin test. Top panel, control subjects; bottom panel, schizophrenic patients. 


\section{DISCUSSION}

Mental stress resulting from the Kraepelin test produced different change patterns of pHVA levels in patients with schizophrenia and control subjects. While a decrease in pHVA concentrations was observed in control subjects, pHVA levels in patients with schizophrenia were not significantly altered by mental stress (Table 1 ). Moreover, the value of changes in pHVA concentrations due to mental stress correlated positively with pre-test pHVA levels in control subjects but not in patients with schizophrenia (Figure 1).

The observed decrease in pHVA levels due to mental stress in control subjects may be explained, at least in part, by changes in the central dopaminergic activity (Sumiyoshi et al. 1998). It has been suggested that the DA turnover in subcortical brain regions such as nucleus accumbens constitutes the major component of the brain HVA output (Csernansky et al. 1990; Lambert et al. 1991). It has also been reported that stress causes a reduction in dopaminergic activity in subcortical nuclei via mechanisms involving increased cortical dopaminergic activity (Friedhoff et al. 1995). Thus, it is possible that the decrease in pHVA concentrations during stress in control subjects was associated with decreased dopaminergic activity in the subcortical area such as nucleus accumbens.

The blunted dopaminergic response to mental stress in schizophrenia, as evidenced by the absence of a decrease in pHVA levels, may be further support for the presence of a DA-dependent restitutive system in the brain (Friedhoff 1985, 1986, 1988; Friedhoff et al. 1995). Together with the lack of a correlation between the baseline pHVA levels and the change values in pHVA levels during mental stress in schizophrenia (Figure 1), the results reported here may indicate that the DA-dependent restitutive system, which normally decreases its own activity in the face of stress, may be disrupted or already down-regulated in schizophrenia, as previously predicted (Friedhoff 1985, 1986, 1988). If cortical and subcortical dopaminergic responses to mental stress is associated with the observed change in pHVA levels in control subjects as discussed above, the results in schizophrenia reported here may have relevance to the suggestion that alterations in the function of frontal cortex and limbic areas result in reduced ability to cope with stress leading to decompensation in schizophrenia (Deutch et al. 1990).

The existence of the DA-dependent restitutive system may reconcile the clinical observation that schizophrenic patients who have low pretreatment dopaminergic activity respond poorly to neuroleptic treatment (Chang et al. 1993; Davis et al. 1985; Green et al. 1993; Sumiyoshi et al. 1997b) with the general view that neuroleptic medication exerts its therapeutic effect by decreasing dopaminergic activity (see Friedhoff 1988 for review).
Several studies report the effect of neuroleptic treatment on pHVA levels. For example, an initial increase in pHVA levels followed by a decrease into the baseline levels has been reported in some patients taking typical neuroleptic drugs such as haloperidol within a relatively short period (4-6 weeks) (Davilia et al. 1988; Green et al. 1993). In some patients receiving neuroleptics studied here, a part of down-regulation of dopaminergic activity may have been achieved by a long-term ( $>6$ months) neuroleptic treatment. However, there was no evidence for differences in pre- or post-test pHVA levels and the change values in pHVA concentrations during stress between neuroleptic-naive patients and those under ongoing neuroleptic treatment. Moreover, in the latter group of patients, no significant correlations were found between these measures of pHVA and neuroleptic doses administered. Although the small samples of our groups require caution for any interpretations, these results suggest that the deficient DA-dependent restitutive system reflected by blunted pHVA response is present in patients with schizophrenia whether or not they are receiving neuroleptics. Further studies including a larger number of neuroleptic-naive subjects combined with psychopathology data would strengthen the clinical significance of the results reported here for the study of schizophrenia.

In conclusion, differential effects of mental stress on pHVA levels between control subjects and patients with schizophrenia were found due to the absence of a decrease in pHVA levels in schizophrenia. The value of changes in pHVA concentrations during stress correlated significantly with pre-test pHVA levels only in control subjects. Although our preliminary study with the small number of subjects limits the conclusions to be drawn, these results may be further support for the presence of a DA-dependent restitutive system in the brain.

\section{ACKNOWLEDGMENTS}

A part of this work was presented at the 21st Congress of Collegium Internationale Neuro-Psychopharmacologicum (CINP) July 14, 1998, in Glasgow, Scotland. The assistance of Dr. Yoshiharu Kawai is deeply appreciated.

\section{REFERENCES}

Akiyama K, Tsuchida K, Kanzaki A, Ujike H, Hamamura T, Kondo K, Mutoh S, Miyanagi K, Kuroda S, Otsuki S (1995): Plasma homovanillic acid levels and therapeutic outcome in schizophrenics: Comparisons of neuroleptic-naive first-episode patients and patients with disease exacerbation due to neuroleptic discontinuance. Biol Psychiatry 38:639-648

Chang W-H, Hwu H-G, Chen T-Y, Lin S-K, Lung F-W, Chen $\mathrm{H}$, Lin W-L, Hu W-H, Lin H-N, Chien C-P (1993): Plasma homovanillic acid and treatment response in a 
large group of schizophrenic patients. Schizophr Res 10: 259-265

Csernansky JG, Barnes DE, Bellows EP, Lombrozo L (1990): Interrelationships between plasma homovanillic acid and indices of dopamine turnover in multiple brain areas during haloperidol and saline administration. Life Sci 46:707-713

Davilia R, Manero E, Zumarraga M, Andia I, Schweitzer JW, Friedhoff AJ (1988): Plasma homovanillic acid as a predictor of response to neuroleptics. Arch Gen Psychiatry 45:564-567

Davis KL, Davidson M, Mohs RC, Kendler KS, David BM, Johns CA, DeNigris Y, Horvath TB (1985): Plasma homovanillic acid concentration and the severity of schizophrenic illness. Science 227:1601-1602

Deutch AY, Clark WA, Roth RH (1990): Prefrontal cortical dopamine depletion enhances the responsiveness of mesolimbic dopamine neurons to stress. Brain Res 521:311-315

Doran A, Picker D, Labarca R, Douillet R, Wolkowitz O, Thomas J, Roy A, Dane S (1985): Evidence for a daily rhythm of plasma HVA in normal controls but not in schizophrenic patients. Psychopharmacol Bull 21:694-697

Friedhoff AJ (1985): Restitutive processes in the regulation of behavior. In Alpert M (ed), Controversies in Schizophrenia: Change and Constancies. New York, Guilford Press, pp 137-144

Friedhoff AJ (1986): A dopamine-dependent restitutive system for the maintenance of mental normalcy. In Burrell CD, Strand FL (eds), Second Colloquium in Biological Sciences. New York, Ann NY Acad Sci 463:860-864

Friedhoff AJ (1988): Dopamine as a mediator of a central stabilizing system. Neuropsycopharmacology 1:189-191

Friedhoff AJ, Carr KD, Uysal S, Schweitzer J (1995): Repeated inescapable stress produces a neuroleptic-like effect on the conditioned avoidance response. Neuropsychopharmacology 13:129-138

Friedhoff AJ, Amin F (1997): Plasma Homovanillic Acid in Schizophrenia: Implication for Presynaptic Dopamine Dysfunction. Washington, DC, American Psychiatric Press

Garver DL, Steinberg JL, McDermott BE, Yao JK, Ramberg JE, Lewis S, Kingsbury SJ (1997): Etiologic heterogeneity of the psychoses: Is there a dopamine psychosis? Neuropsychopharmacology 16:191-201

Green AI, Alam MY, Boshes RA, Waternaux C, Pappalardo KM, Fitzgibbon ME, Tsuang MT, Schildkraut JJ (1993): Haloperidol response and plasma catecholamines and their metabolites. Schizophr Res 10:33-37

Kopin IJ, Bankiewicz KS, Harvey-White J (1988): Assessment of brain dopamine metabolism from plasma HVA and MHPG during debisoquin treatment: Validation in monkeys treated with MPTP. Neuropsychopharmacology 1:119-125

Koreen AR, Lieberman J, Alvir J, Mayerhoff D, Loebel A, Chakos M, Amin F, Copper T (1994): Plasma homovanillic acid levels in first-episode schizophrenia. Arch Gen Psychiatry 51:132-138

Kuraishi S, Kato M, Tsujioka B (1957): Development of the "Uchida-Kraepelin psychodiagnostic test" in Japan. Psychologia 1:104-109
Lambert GW, Eisenhofer G, Cox HS, Horne M, Kalff V, Kelly M, Jennings GL, Esler MD (1991): Direct determination of homovanillic acid release from the human brain, an indicator of central dopaminergic activity. Life Sci 49: 1061-1072

Lambert GW, Eisenhofer G, Jennings GL, Esler MD (1993): Regional homovanillic acid production in human. Life Sci 53:63-75

Maas JW, Contreras SA, Miller AL, Berman N, Bowden CL, Javor MA, Seleshi E, Weintraub S (1993): Studies on catecholamine metabolism in schizophrenia/psychosis-1. Neuropsychopharmacology 8:97-109

Maas JW, Contreras SA, Seleshi E, Bowden CL (1988): Dopamine metabolism and disposition in schizophrenic patients: Studies using debrisoquin sulfate. Arch Gen Psychiatry 45:553-559

Nagaoka S, Iwamoto N, Arai H (1997): First-episode neuroleptic-free schizophrenics: Concentrations of monoamines and their metabolites in plasma and their correlations with clinical responses to haloperidol treatment. Biol Psychiatry 41:857-864

Pickar D, Labarca R, Linnoila M, Roy A, Hommer D, Everett D, Paul SM (1984): Neuroleptic-induced decrease in plasma homovanillic acid and antipsychotic activity in schizophrenic patients. Science 225:954-957

Reynolds GP (1983): Increased concentrations and lateral asymmetry of amygdala dopamine in schizophrenia. Nature 305:527-529

Seeman P, Guan H-C, Van Tol HHM (1993): Dopamine D4 receptors elevated in schizophrenia. Nature 365:441-445

Steinberg JL, Graver DL, Moeller FG, Raese JD, Orsulak PJ (1993): Serum homovanillic acid levels in schizophrenic patients and normal control subjects. Psychiatry Res 48:93-106

Sumiyoshi T, Hasegawa M, Jayathilake K, Meltzer HY (1997a): Sex differences in plasma homovanillic acid levels in schizophrenia and normal controls: Relation to neuroleptic resistance. Biol Psychiatry 41:560-566

Sumiyoshi T, Hasegawa M, Jayathilake K, Meltzer HY (1997b): Prediction of short-term changes in symptom severity by baseline plasma homovanillic acid levels in schizophrenic patients receiving clozapine. Psychiatry Res 69:113-121

Sumiyoshi T, Yotsutsuji M, Kurachi M, Itoh H, Kurokawa K, Saitoh O (1997c): Effect of mental stress on plasma homovanillic acid levels in healthy subjects. Soc Neurosci Abstr 23:2036

Sumiyoshi T, Yotsutsuji T, Kurachi M, Itoh H, Kurokawa K, Saitoh O (1998): Effect of mental stress on plasma homovanillic acid in healthy human subjects. Neuropsychopharmacology 19:70-73

Whelton CL, Gupta RN, Cleghorn JM, Ballagh SR (1993): Influence of renal clearance on peripheral homovanillic acid measurements in healthy subjects and schizophrenic patients. Schizophr Res 11:33-40

Wong DF, Wagner HN, Tune LE, Dannals RF, Pearlsson GD, Links JM, Tamminga CA, Broussolle EP, Ravert HT, Wilson AA, Toung JKF, Malat J, Williams FA, O'Touma LA, Snyder SH, Kuhar MJ, Gjedde A (1986): Positron emission tomography reveals elevated D2-dopamine receptors in drug-naive schizophrenia. Science 234:1558-1563 